


\title{
Utilización eficaz de las tecnologías de información y comunicación en procesos sancionatorios y responsabilidad fiscal
}

\author{
DOI: https://doi.org/10.46398/cuestpol.3969.02
}

Angélica Gavidia Pacheco *

Eder Alberto Molina Araujo **

Ingrid Julissa Illidge Correa ***

Leonardo de Jesús Díaz ****

Indira Yulieth Illidge Correa ${ }^{* * * *}$

\section{Resumen}

El objetivo del artículo fue discutir la relación entre las tecnologías de la información ante los procesos sancionatorios y responsabilidad fiscal, sucedidos en los órganos de control fiscal territorial, espacios en los cuales se requiere mayores recursos en tecnología con destinación específica para ello, aprovechando el momento de la vigencia del Acto Legislativo 04 de 2019 en Colombia. En lo metodológico se hizo uso del diseño documental de investigación próximo al ensayo crítico. Para ilustrar los argumentos y puntos de vista del equipo de investigación, se muestra situaciones en las cuales se podría mejorar el proceso administrativo sancionatorio y de responsabilidad fiscal, enfatizando en la hipótesis que la utilización de tecnologías de la información permite el afianzamiento al derecho fundamental del debido proceso de los investigados. Se concluye que, cuando se extienden las herramientas tecnológicas a la institucionalidad del Estado Colombiano se logra tener un mejor acercamiento con la ciudadanía, lo cual genera una expectativa positiva en la satisfacción de sus necesidades, siendo la esencia del Estado Social de Derecho construir en todo momento una democracia de resultados que transcienda a las formalidades procesales y amplia la justicia social.

* Abogada, especialista en derecho penal, Magister en Educación y Magíster en Ciencias Políticas, Doctor ante en Ciencias económicas y administrativas. Alcaldía Distrital de Barranquilla. ORCID ID: https:// orcid.org/oooo-0002-8436-6389. Email: angelicagavidiapacheco@hotmail.com

** Abogado, especialista en Derecho Administrativo, consultor de proyectos. Contraloría Distrital de Barranquilla. ORCID ID: https://orcid.org/0000-0002-0851-7763. Email: edermolina28@hotmail.com

*** Abogada, especialista en Derecho de Familia, Gestión Pública, Laboral y Seguridad Social y Doctora en Ciencias Económicas y Administrativas. Universidad Simón Bolívar. ORCID ID: https://orcid.org/0000-0001-5129-4326. Email: ingridillidge@hotmail.com

**** Abogado, especialista en Gestión Pública, Magister en Derecho Administrativo. Universidad Nacional abierta y a Distancia. ORCID ID: https://orcid.org/0000-0002-2061-438X. Email: leonardodiaz1121@hotmail.com

$5 * * * * *$ Administradora de Empresas y Contadora Pública, Magister en Gestión de Administración de Organizaciones. Universidad Nacional Abierta y a Distancia. ORCID ID: https://orcid.org/0000-0001-89057668. Email: ingridillidge@hotmail.com 
Palabras clave: derecho y TICS; debido proceso; proceso sancionatorio; responsabilidad Fiscal; tecnologías de la información en Colombia.

\title{
Effective use of information and communication technologies in sanctioning processes and fiscal responsibility
}

\begin{abstract}
The objective of the article was to discuss the relationship between information technologies in the face of the sanctioning processes and fiscal responsibility, which occurred in the territorial tax control bodies, spaces and which more resources are required in technology with specific purpose for this purpose, taking advantage of the moment of the validity of Legislative Act 04 of 2019 in Colombia. Methodologically, the documentary research design near the critical essay was used. To illustrate the arguments and views of the research team, it shows situations in which the administrative process of sanctioning and fiscal responsibility could be improved, emphasizing the hypothesis that the use of information technologies allows the strengthening of the fundamental right of due process of those investigated. It is concluded that, when technological tools are extended to the institutionality of the Colombian State, it is possible to have a better approach with citizenship, which generates a positive expectation in meeting their needs, being the essence of the Social State of Law to build at all times a democracy of results that transcends procedural formalities and expands social justice.
\end{abstract}

Keywords: law and ICT; due process; sanctioning process; Fiscal responsibility; information technologies in Colombia.

\section{Introducción}

La utilización de las tecnologías de la información (TIC) en el desarrollo de procesos administrativos sancionatorios y de responsabilidad fiscal constituye en elemento fundamental de garantías constitucionales en el debido proceso y publicidad, reflejando el compromiso del Estado colombiano en la búsqueda de acercamientos más agiles que permitan una mejor relación Estado-Ciudadanía. En este sentido, si el Estado tiene facultad sancionatoria y de responsabilidad a través de los entes nacionales 
Angéliça Gavidia Pacheco, Eder Alberto Molina Araujo, Ingrid Julissa Illidge Correa, Leonardo de Jesús Díaz y Indira Yulieth Illidge Correa

y territoriales de control fiscal, éstos últimos que se mantienen vigentes en virtud del Acto legislativo 04 de 2019, el cual plantea expresamente el fortalecimiento del control fiscal.

Este derecho es una manifestación de poder jurídico necesaria para la regulación de la vida en sociedad y para que la administración pueda cumplir adecuadamente sus funciones. Aunque se ejercita a partir de la vulneración o perturbación de reglas preestablecidas, tiene una cierta finalidad preventiva en el simple hecho de proponer un cuadro sancionador como consecuencia del incumplimiento de las prescripciones normativas. Se traduce normalmente en la sanción correctiva y disciplinaria para reprimir las acciones u omisiones antijurídicas y constituye además un complemento de la potestad de mando, pues contribuye al cumplimiento de las decisiones administrativas.

Es así como, al menos en teoría, el poder punitivo que se reconoce al Estado encuentra fundamento en la exigibilidad de las normas generales y no en una atribución de privilegio a favor de determinadas personas, sectores o partidos políticos. La unidad del derecho punitivo del Estado se vigoriza en el ejercicio de sus atribuciones de reparto funcional, jurisdiccional y administrativo. En consecuencia, es absolutamente imposible pretender un Estado intervencionista sin mecanismos sancionatorios. La vigilancia y la inspección se tornarían inoperantes, si faltara el elemento coercitivo con base a un poder vinculante.

El derecho al debido proceso como mecanismo efectivo de protección al derecho a la defensa y contradicción el cual debe permitir la generación de garantías para el ciudadano frente al poder del Estado, tomando como referencia la concepción del Estado Social de Derecho que la Constitución de 1991 consagra en el régimen colombiano, los objetivos proclamados en el preámbulo, así como la existencia de derechos económicos fundamentales, son elementos que tienen implicaciones sustantivas sobre la legitimidad constitucional de las políticas económicas.

Es así como en este artículo se busca determinar si la utilización de las tecnologías de la información permiten la garantía del derecho al debido proceso y, al mismo tiempo, posibilitan la mejora en las organización, teniendo en cuenta que: "La eficiencia interna se verá directamente impactada, puesto que los procesos de automatización de tareas mecánicas y el establecimiento de canales de comunicación vía web, permitirá liberar a trabajadores de tareas repetitivas y de poco valor añadido" (Aibar, 2001: $\mathrm{s} / \mathrm{p})$.

Por lo demás:

El período de tiempo durante el cual tiene lugar una innovación en la tecnología de la información se convierte en la fuerza latente de la transformación social, capaz de acarrear una expansión en la calidad y en la cantidad de información y un aumento en gran escala del almacenamiento de la información (Masuda, 1984: 67). 


\section{Planteamiento del problema}

Puede indicarse que la utilización de herramientas tecnológicas de la información es un deber de las autoridades para la generación de garantías constitucionales como el debido proceso, entendiendo que: "El conjunto de tecnologías permiten el acceso, producción, tratamiento y comunicación de información presentada en diferentes códigos (texto, imagen, sonido)" (Muñoz, 2016:11). Por regla general, estas herramientas permiten la generación de garantías a los implicados en una investigación, así como tiene efectos en el sistema de gestión ambiental, la adopción de buenas prácticas en el desarrollo de actividades para mejorar impactos ambientales generados en el uso del papel.

Por ello, la administración pública está en el deber de prestar sus servicios de forma que permita a cualquier ciudadano conocer la información, pues ésta "debe ofrecer una cierta flexibilidad tecnológica para que el mayor número de ciudadanos pueda relacionarse con ella" (Guillén, 2010: 52). Sin embargo, existen falencias en entidades públicas que desarrollan procesos administrativos sancionatorios en la utilización de herramientas tecnológicas de la información para la generación de garantías constitucionales como el debido proceso. De modo que es la ciudadanía requiere del esfuerzo de los representantes de la administración a efectos de soluciones los graves problemas de comunicación, ofreciendo ésta última las herramientas necesarias para lograr esa comunicación fluida, por los medios disponibles tecnológicos.

Muchas entidades no han podido generar alternativas de virtualidad en el desarrollo de sus procesos administrativos sancionatorios, muy a pesar de que el principio de necesidad de la prueba les exige acopiar todos los medios de convicción posibles, para luego, tomar la decisión que corresponda reconociendo el mérito probatorio de cada medio en particular, y de todos en conjunto, en la esfera del principio de la unidad de la prueba. Es por ello, que la tecnología facilita la asunción de garantías democráticas a la ciudadanía.

Ahora bien, se ha podido determinar que no existe una norma reglamentaria que permita a los conductores realizar sus descargos en cualquier lugar donde se encuentren, a través de las herramientas virtuales de información, que se han venido implementando en otras áreas jurídicas como el derecho penal. Esto necesario, en aras de la materialización del derecho al debido proceso.

Ello implica la aplicación de una forma de responsabilidad objetiva que, en el derecho sancionatorio está proscrita por la Constitución Política en su artículo 29. Es así como: 
Angélica Gavidia Pacheco, Eder Alberto Molina Araujo, Ingrid Julissa Illidge Correa, Leonardo de Jesús Díaz y Indira Yulieth Illidge Correa

Lo que se pretende con la implementación del procedimiento administrativo electrónico es el acercamiento dela administración hacialos ciudadanos, de manera que el principio de igualdad en el desarrollo de la administración electrónica será fundamental para concretar sus principales postulados (Muñoz, 2016: 21).

Es un deber de las autoridades velar porque la implementación de esos instrumentos en sus relaciones con los ciudadanos se haga de una manera equilibrada (Sánchez, 2014). En la actualidad las autoridades deben atender:

La brecha digital como uno de los principales desafíos que deberá asumir la administración en el proceso de implementación y desarrollo de las TIC, pues se trata de un nuevo tipo de desigualdad para aquellos que no tienen o que tienen un acceso limitado a la red, así como para los que no son capaces de sacarle partido (Castells, 2001: 275).

El conocimiento del problema de investigación nos llevó a formular la pregunta siguiente ¿Con la implementación de herramientas de la información y las comunicaciones en las audiencias ante los órganos de control fiscal territorial, se permite el afianzamiento al derecho fundamental al debido proceso de los investigados?

\section{Análisis y discusión de resultados}

La nueva era de la información se ha generado desde el auge de las matemáticas como modelo de racionamiento que da lugar al surgimiento de la era industrial y científica basada en áreas como la estadística, que permitieron organizar los estados nación, para más adelante dar paso a la aparición de las máquinas informáticas y su estrecha relación con la investigación al servicio de las operaciones militares. Ya iniciados los años sesenta, y ante el advenimiento de la sociedad post-industrial, Matterlart (2001) refiere que el eje central de la sociedad radica en la expansión de los servicios humanos y, sobre todo, la inflación de servicios técnicos y profesionales

Con la Cumbre Mundial sobre la Sociedad de la Información (CMSI), realizada en dos fases, se concretan los fundamentos de la Sociedad de la Información con la intención de fomentar la utilización de las TIC en la construcción de una sociedad integradora y orientada al desarrollo equitativo de las naciones (Unión Internacional de Telecomunicaciones).

Como referentes teóricos a la implementación de estrategias probatorias de las Tecnologías de Información se remontan a la aparición del modelo de la Nueva Gestión Pública. Este modelo de gestión que surgió no se refiere a la forma del poder público; sino a la efectividad y transparencia de la gestión pública, estableciendo la primacía de ciertos principios y mecanismos 
que garanticen ese cometido, entre los cuales, encontramos lo que reza taxativamente el Artículo 209 de la Constitución Política Colombiana:

\begin{abstract}
La Función administrativa está al servicio de los intereses generales y se desarrolla con fundamento en los principios de igualdad, moralidad, eficacia, economía, celeridad, imparcialidad y publicidad, mediante la descentralización, la delegación y la desconcentración de funciones. Las autoridades administrativas deben coordinar sus actuaciones para el adecuado cumplimiento de los fines del Estado. La administración pública, en todos sus órdenes, tendrá un control interno que se ejercerá en los términos que señale la ley (Constitución Pólitica Colombiana, 1991)
\end{abstract}

Al decir de Moyado (2011), la tradicion anglosajona que sirve de paradigma en estos temas presenta a la Nueva Gestión Pública como un enfoque de transición de la vieja administración pública poniendo ahora el acento en los resultados y en la responsabilidad, en organizaciones, recursos y condiciones contractuales más flexibles y en técnicas de evaluación del rendimiento de los recursos humanos.

La aplicabilidad del modelo de la Nueva Gestión Pública en los mecanismos de "reforma" del Estado Colombiano se ha implementado principalmente en el sistema de contratación pública, ya que sirve de soporte tanto en el mejoramiento de la gestión pública como en la prestación de servicios públicos. Para poder cumplir este cometido se deben mejorar los sistemas de información en materia de normativas vigentes y de publicidad de los procesos contractuales en curso, garantizando de este modo la transparencia en la gestión contractual.

Colombia ha dado un paso importante a la disposición de canales de acceso para la ciudadanía entre los que encontramos Gobierno digital, modelo de organización política que busca integrar las nuevas tecnologías en la administración pública para incrementar sustancialmente su eficacia y eficiencia. Se espera con esta estrategia que todas las entidades públicas dispongan de un canal web donde la ciudadanía pueda realizar trámites y consultas, para que la comunidad pueda participar activamente en la planeación, ejecución y control, de las diferentes actividades, entre ellas la de la contratación pública.

Señala Nieto (1994) que en el derecho sancionatorio administrativo juega un papel importante el principio de publicidad al decir que: Desde el punto de vista de la política sancionadora se exige, además su divulgación, más o menos larga y detallada según sea el grado de especialización o profesionalización de sus destinatarios. Señala el mismo autor que el auge de la teoría de la potestad administrativa sancionatoria busca generar mayor presencia administrativa del estado en el juzgamiento de una determinada conducta, contrario al lento y desgastante poder punitivo jurisdiccional del estado; es la reducción de la intervención judicial, bajo 
Angéliça Gavidia Pacheco, Eder Alberto Molina Araujo, Ingrid Julissa Illidge Correa, Leonardo de Jesús Díaz y Indira Yulieth Illidge Correa

razones de conveniencia y en aras de unos procedimientos más agiles y eficaces que consulten la buena marcha del complejo estatal, este es el reto de la administración pública.

La mayor presencia administrativa del Estado en el juzgamiento de una determinada conducta, en contraste con el severo poder punitivo jurisdiccional del mismo, hace resaltar el principio de la mínima intervención penal. La despenalización de conductas señaladas en el derecho penal, realiza modificaciones sustantivas y procesales, así el derecho de tránsito y transportes cuenta con sus propias normas sustantivas y procesales, alternando competencias, lo que obliga al Estado a establecer una reestructuración no solo del procedimiento sancionatorio y del proceso jurisdiccional, sino también de la delimitación jurídica de cada infracción y de la dosificación de la sanción respectiva, es decir de la tipificación de la ilicitud y la pena.

En los nuevos procedimientos legales de las ramas del derecho urge la implementación de las mínimas garantías penalistas que rodean el entorno procesal, por lo tanto, los aspectos modernos que impliquen mejorías en el procedimiento para imposición de sanciones fundamentales para el derecho administrativo sancionatorio se deben adoptar las mejores alternativas en aras de la protección y defensa a los derechos y garantías fundamentales.

Esta imposición de sanciones debe desarrollarse conforme a los fundamentos constitucionales, y al derecho a la defensa como elemento fundante del debido proceso en derechos como tránsito, sancionatorios ante entes de control, entidades que realicen cobro coactivo en cualquier orden nacional o territorial. Bajo esta perspectiva se puede hablar de un derecho administrativo sancionador en la medida en que la potestad punitiva del Estado se impulse dentro de un ordenamiento propio que clarifique las normas procesales, tipifique la falta, precise la dosificación punitiva, reserve legalmente la transgresión y de todas formas consagre u mínimo de garantías.

Para Garcia (2014), estas garantías pueden generarse a través de la administración electrónica, gobierno digital o gobierno abierto que son simplemente neologismos que describen el uso de las tecnologías digitales para mejorar el acceso y la divulgación de la información y los servicios públicos, así como la relación ciudadanos-gobiernos. Con el objeto de promover la transparencia, y como consecuencia, la colaboración y participación de ciudadanos y administraciones públicas de una manera más justa en la sociedad.

La tecnología puede considerarse autónoma y determinista cuando las normas mediante las cuales progresa se eliminan del discurso político y ético y cuando los objetivos de la eficiencia o de la productividad se convierten en sustitutos de los debates sobre los métodos, las alternativas, los medios y los fines basados en valores (Habermas, 1970: 58). 
En efecto, las nuevas tecnologías posicionaron a la información como el principal activo del siglo XXI, permitieron la interconexión de seres humanos, superando las barreras de tiempo y espacio y, simultáneamente, demostraron que el conocimiento y la inteligencia son las mayores riquezas de la humanidad; por ello la competitividad en el ser humano creador de organizaciones en la actualidad se basa en la correcta utilización de los recursos que tiene el entorno, de la cual las herramientas tecnológicas son vitales para su crecimiento y desarrollo, pues utilizadas en su debida forma repercuten en la productividad y desarrollo de la empresa. Por lo tanto:

La tecnología ejerce una influencia causal en la práctica social, donde a la luz de la situación pasada y actual del desarrollo tecnológico y de las leyes de la naturaleza, el cambio social no puede seguir en el futuro más que un único curso posible, es decir, que las estructuras sociales evolucionan adaptándose al cambio tecnológico, independientemente de lo que pensaran o desearan los individuos (Bimber, 1994: 99-100).

Las tecnologías de la información y las comunicaciones juegan un papel determinante en el proceso de modernización del Estado:

Tiene como objeto garantizar la efectividad de los principios de la función administrativa como son, la eficacia, la economía y la celeridad; de igual forma los referidos medios constituyen un instrumento razonable y justificado para el cumplimiento de los fines estatales y su uso por parte de la administración pública obedece a la necesidad de estar acorde con las exigencias actuales en torno a la inmersión del Estado en la sociedad de la información y el conocimiento (Consejo de Estado, 2011: 01).

De igual manera la masificación de la información es destacada por el legislador colombiano al reconocer la importancia del principio de igualdad en el acceso a las TIC para ejercer el derecho a relacionarse con la administración, reiterando en el artículo 53 de la Ley 1437 de 2011 que: "Para garantizar la igualdad de acceso a la administración, la autoridad deberá asegurar mecanismos suficientes y adecuados de acceso gratuito a los medios electrónicos, o permitir el uso alternativo de otros procedimientos" (Congreso de la República, 2011, Ley 1437, art. 53). Con base a este artículo 53 se permite que los procedimientos administrativos podrán realizarse a través de medios electrónicos, siempre y cuando se garantice la igualdad en el acceso a los mismos, reconoce las dificultades en materia de acceso y uso de TIC y la brecha social y cultural de nuestra sociedad.

La entidad pública incluido los organismos de control deben contar con un sistema de seguridad de información que permita la generación de garantía del derecho a la buena fe, pues la aplicación de este principio a los procedimientos administrativos se concreta en que la administración 
Angélica Gavidia Pacheco, Eder Alberto Molina Araujo, Ingrid Julissa Illidge Correa, Leonardo de Jesús Díaz y Indira Yulieth Illidge Correa responsabilidad fiscal

tiene derecho recibir, de parte de los administrados, la información veraz y completa que sea necesaria para la adopción de la decisión; los sujetos del procedimiento deben actuar de tal manera que no se dilate innecesariamente la actuación administrativa; el administrado tiene derecho a confiar en que la administración adoptará una decisión que respeta el bloque de legalidad al cual se encuentra sometida, y el procedimiento debe adelantarse no sólo con plena sujeción a las reglas de trámite sino con especial esmero para que efectivamente se logre la garantía de los derechos de quienes actúan dentro del mismo.

En suma, la buena fe dentro del procedimiento administrativo se concreta en que el deber de los sujetos de guardar un comportamiento leal y honesto dentro de su trámite, en que la administración proferirá una decisión ajustada a derecho y en que los administrados no ocultarán informaciones ni entregarán informaciones falsas. En este sentido, el procedimiento administrativo electrónico:

Se sustenta en un modelo que, basado en el uso de las tecnologías de la información y las comunicaciones, pretende mejorar la tramitación de los procedimientos administrativos, aumentando la eficiencia de la gestión interna, ganando en celeridad y transparencia, intentado obtener resultados más uniformes y económicos y, en último término, otorgando un mejor servicio a los ciudadanos. De esta forma, abarca distintos ámbitos de acción, de una parte, la atención al ciudadano e interesado, a través del uso de plataformas electrónicas para interactuar con las instituciones respectivas, y, de otra, aquellas asociadas al mejoramiento de la administración interna del procedimiento (Moya, 2008: 208).

Una de las maneras más nítidas para clarificar la importancia de la utilización de las tecnologías de la información se encuentra en el acto administrativo contenido en el formato documento electrónico, evidentes frente al acto tradicional de papel, por lo siguiente:

1. El soporte electrónico permite mayor durabilidad en el tiempo.

2. El soporte electrónico da mayor seguridad e inalterabilidad que el documento papel.

3. El soporte electrónico permite insertarlo en la Internet, dotándolo de mucha mayor publicidad que el papel.

4. El soporte electrónico permite imprimirle una firma electrónica, la cual es más segura que la firma autógrafa.

5. El soporte electrónico permite multicopiarlo tantas veces se quiera a menores costos y;

6. En general, el soporte electrónico no necesita de materialización alguna, pero sigue sirviendo de constancia, soportey, en consecuencia, 
es consultable, revisable, comunicable, inmodificable, autenticable (Laguado, 2003).

Es así como la notificación electrónica permite que:

Las autoridades podrán notificar sus actos a través de medios electrónicos, siempre que el administrado haya aceptado este medio de notificación. Sin embargo, durante el desarrollo de la actuación el interesado podrá solicitar a la autoridad que las notificaciones sucesivas no se realicen por medios electrónicos (Congreso de la República, 2011, Ley 1437, art. 56).

Pues, en otras razones:

El imponer la comunicación entre la administración y los administrados, solamente, a través de medios electrónicos puede resultar discriminatorio, por lo tanto, se reconoce en la norma en comento que "no todas las personas residentes en el territorio nacional cuentan con los medios materiales y, sobre todo, con el conocimiento tecnológico para que los procedimientos sean plenamente electrónicos; de aquí que se mantengan los escritos y se incorporen los orales, y con ellos simultáneamente juegue y actúe el aparato administrativo del Estado (Santofimio-Gamboa, 2011: 205).

Ahora conviene preguntar entonces ¿Qué puede hacerse la contraloría si no puede agotarse la notificación personal o por aviso y es devuelta la comunicación? En este caso es válida la comunicación electrónica en algún correo que se tenga de la persona o en la publicación en página web, teniendo presente que la entidad debe tener este procedimiento y algún manual, resolución y/o en su esquema de publicación, actuando conforme a lo establecido en la Ley 1712 de 2014, hecho éste que se encuentra amparado por la jurisprudencia constitucional.

Por ello, la (Corte Constitucional, 2013, Sentencia C-012) ha señalado que la publicación por aviso en la página web de la Dirección de Impuestos y Aduanas Nacionales (DIAN) y en un lugar de acceso al público de la misma entidad cuando son devueltas las notificaciones por correo, no desconoce el debido proceso, el orden justo ni el deber de garantizar los derechos de las personas, ya que la previsión legal de este mecanismo de notificación:

1. Es desarrollo de la potestad de configuración legislativa --en este caso, extraordinaria-- en materia de procedimientos administrativos y del deber constitucional de contribución a la financiación de los gastos del Estado y la sociedad y;

2. No significa un ejercicio arbitrario o desproporcionado de tal potestad de configuración de los procedimientos administrativos y de la notificación de las actuaciones de la administración, ya que solo se activa, como mecanismo subsidiario. (Corte Constitucional, 2013, Sentencia C-012) 
Angélica Gavidia Pacheco, Eder Alberto Molina Araujo, Ingrid Julissa Illidge Correa, Leonardo de Jesús Díaz y Indira Yulieth Illidge Correa

Sin embargo, también debe tenerse en cuenta que se pueden dilatar los plazos, en cuyo caso la eficacia de los actos administrativos quedaría sujeta a disposición de los intereses del destinatario, máxime, cuando en el articulado no se exige una carga para el administrado teniendo en cuenta que su precedente aceptación de este medio de notificación no debe significar mayor controversia suponiendo:

(...) el caso en el que el interesado invoque a su favor la no recepción del documento, pues para el efecto lo que se pone en duda es la confianza depositada en los proveedores de servicios que intermedian en el proceso de certificación de emisión y recepción del acto de la administración (Corte Constitucional, 2000: Sentencia C-662).

De igual manera, también se permite la interposición de:

Recursos contra los actos administrativos, aún los de carácter disciplinario, pueden ser válidamente interpuestos a través del correo electrónico, siempre que se presenten de manera oportuna y se cumplan los demás requisitos previstos en las normas aplicables (Corte Constitucional, 2013: Sentencia C-286).

Por otro lado, la Sentencia T-145 de 1993 de la Corte Constitucional estableció que la imposición de sanciones o medidas correccionales, incluidos los procesos de responsabilidad fiscal debe sujetarse a las garantías procesales del derecho de defensa y contradicción; en consecuencia, carece de respaldo constitucional la imposición de sentencias administrativas de plano con fundamento en la comprobación objetiva de una conducta ilegal, en razón del desconocimiento que ello implica de los principios de contradicción y de presunción de inocencia, los cuales hacen parte del núcleo esencial del derecho al debido proceso.

Del mismo modo, en Sentencia T-582 de 1992 emanada de la Corte Constitucional esta señaló que: "Toda persona tiene derecho que antes de ser sancionada, se lleve a cabo un procedimiento mínimo que incluye la garantía de su defensa". Por lo tanto, conforme a las anteriores providencias, no es el culto a la forma ni reverencia a la estructura extrema lo que hace del procedimiento su justificación, sino la misma ética administrativa y lo que, es más, el respeto a los derechos fundamentales del individuo.

Con base a la pregunta problema se puede indicar que el proceso administrativo sancionatorio $\mathrm{y}$, de responsabilidad fiscal, no pueden desconocer las garantías constitucionales en especial el debido proceso definido como la regulación jurídica que de manera previa limita los poderes del Estado y, establece las garantías de protección a los derechos de los administrados, de modo que ninguna de las actuaciones de las autoridades públicas depende de su propio arbitrio, sino que se encuentran -en todos los casos- sujetas a los procedimientos señalados en la ley. 
Debe enfatizarse en que la utilización de herramientas tecnológicas en procesos administrativos sancionatorios permite la garantía del debido proceso al consentir herramientas para probar hechos y evitar el cumulo de papel, cuestión que está acorde a una realidad contemporánea donde la tecnología hace parte integral de la cotidianidad; a su vez, se posibilita la carga dinámica de las pruebas en el sentido de permitir probar hechos con medios tecnológicos, lo cual, con base a la equivalencia funcional es legal, tomando como referencia que la carga probatoria no es una obligación ni un deber, por no existir sujeto o entidad legitimada para exigir su cumplimiento. Tiene necesidad que aparezca probado el hecho la parte que soporta la carga, pero su prueba puede lograrse por la actividad oficiosa del juez o de la contraparte (Parra, 2004).

Según el Consejo de Estado (2012), La administración electrónica se desarrolla en tres dimensiones: primero, la prestación de servicios (e-administración), que se refiere a la gestión, información, tramitación de servicios y formulación de quejas y sugerencias; en segundo lugar, la promoción de la democracia (e-democracia), que implica el fortalecimiento de una nueva relación política gobierno-ciudadanía, la consulta y generación de reclamos, el logro de la participación, deliberación en la toma de decisiones públicas y la fiscalización de la labor pública por parte de los ciudadanos, a través de la exigencia de la transparencia y de rendición de cuentas y; por último, la motivación en la elaboración de políticas públicas (e-gobernanza), que se caracteriza por la participación activa en el diseño, gestión, implementación y evaluación de políticas públicas.

En efecto, la Ley 1341 de 2009 por medio de la cual se señala en su artículo No. 6 que las: “(...) Tecnologías de la Información y las Comunicaciones (en adelante TIC), son el conjunto de recursos, herramientas, equipos, programas informáticos, aplicaciones, redes y medios; que permiten la compilación, procesamiento, almacenamiento, transmisión de información como: voz, datos, texto, video e imágenes.

La implementación de las herramientas tecnológicas y virtuales de comunicación (TIC), constituyen medidas necesarias para el mejoramiento de las audiencias que se lleven a cabo, por ejemplo, ante las autoridades de tránsito, con el propósito de una mejor materialización al derecho a la defensa significa una propuesta que se considera viable para ser implementada en el marco jurídico colombiano. Por lo que "la eficiencia, en resumen, como motor interno de la innovación tecnológica, se interpreta como un factor puramente técnico (o científico), objetivo, indiscutible y al margen de cualquier consideración social o valorativa” (Aibar, 2001: 3), siendo una medida alternativa de eficiencia administrativa la búsqueda de soluciones tecnológicas para satisfacer las necesidades de la población, habida de soluciones, más allá de las formalidades. 
Angélica Gavidia Pacheco, Eder Alberto Molina Araujo, Ingrid Julissa Illidge Correa, Leonardo de Jesús Díaz y Indira Yulieth Illidge Correa

Utilización eficaz de las tecnologías de información y comunicación en procesos sancionatorios y responsabilidad fiscal

Las tecnologías de la información además garantizan:

La finalidad de la moralidad dirigida a dotar de transparencia y claridad los procedimientos a través de los cuales actúa la administración. Es en este aparte donde las TIC cobran importancia, como quiera que estas herramientas simplifican los trámites, aceleran los tiempos de respuesta, acercan al ciudadano con la administración, etc., aspectos todos que contribuyen a la legitimación del orden constitucional (Corte Constitucional, 1996: Sentencia C-319).

De esta manera se permite un acercamiento con el ciudadano, teniendo en cuenta que el poder soberano es delegado en varios de sus miembros para que lo representen y, es por el pueblo, al que deben garantizar sus derechos en virtud de ese poder delegado; por ello, se requieren de servidores públicos con competencias funcionales y vocación de servicio, que propicien niveles de acercamiento entre la sociedad y la administración estatal en las entidades territoriales que permiten prever mejoras en la legitimidad y el reconocimiento de las instituciones públicas.

De igual manera, fomentar la utilización general de las tecnologías de la información en la administración pública garantiza el principio de eficacia, por lo que:

En desarrollo de este principio se busca que la administración mejore notablemente la gestión pública garantizando a los usuarios el derecho a un trámite o procedimiento sin dilaciones indebidas e injustificadas apoyando y facilitando la interacción del ciudadano y la administración a través de medios no presenciales, para lo cual las TIC serán la herramienta que permita optimizar y estandarizar los procesos y procedimientos internos de las entidades (Ministerio de Comunicaciones, 2000, Documento Conpes 3072).

Además "Resulta indispensable para el desarrollo efectivo del derecho a relacionase electrónicamente con la administración” (Muñoz, 2014: 46). Por tal razón, es fundamental que las autoridades administrativas presten mayor atención, no solo a la implementación de una sede electrónica, sino a garantizar las condiciones de calidad, seguridad, disponibilidad, accesibilidad, neutralidad e interoperabilidad, consagradas en el artículo 60 del CPACA En la administración electrónica, también se garantiza el principio de economía al coadyuvar con la racionalización de gastos en la administración pública, a lo cual se debe privilegiar el uso delas herramientas tecnológicas. Por lo tanto, el tiempo tomado por la administración de justicia en adoptar decisiones intermedias o definitivas, en los distintos procesos judiciales es determinante. En el artículo 29 de la Constitución Política se establece que las dilaciones injustificadas son violatorias del derecho al debido proceso y, el artículo 228 de la Carta Magna dispone que los términos procesales se observarán con diligencia y su incumplimiento 
será sancionado, aunque la realidad es otra. La justicia como un principio moral que inclina a obrar y juzgar respetando la verdad y dando a cada uno lo que le corresponde, como pilar fundamental en el ordenamiento jurídico, debería ser aplicada con celeridad por el Estado, quien es el encargado de velar por los derechos e intereses de la sociedad.

Desde el punto de vista de las tecnologías de la información y las comunicaciones, estos dos mandatos constitucionales configuran la estrategia rectora del e-government, porque en cualquier etapa o nivel de presencia oficial en la red, la información que se publique se debe mantener veraz, íntegra, actualizada y completa; de lo contrario, la estrategia pierde credibilidad y los usuarios preferirán utilizar canales tradicionales de información.

Las tecnologías de la información permiten la generación de garantías en los principios de publicidad y transparencia, en la cual estos principios constituyen la esencia del procedimiento administrativo democrático. $\mathrm{Al}$ referirse a ellos se alude a la necesidad de que las actuaciones y decisiones de las autoridades sean públicas y abiertas a la totalidad de los asociados.

El principio de transparencia implica que los administrados pueden, en ejercicio del derecho de acceso a la información, conocer la actividad de la administración, pues dicha prerrogativa "genera la obligación correlativa de divulgar de modo proactivo la información pública y responder de buena fe, de manera adecuada, veraz, oportuna y accesible a las solicitudes, lo que conlleva la obligación de producir o capturar información pública (Sánchez, 2014: 98).

En el mismo orden de ideas, debe enfatizarse que:

Si bien resulta excesivo afirmar que la tecnología per se implica eficiencia en las actuaciones de la administración, es innegable que la racionalidad técnica está impactando notablemente las relaciones humanas y transformando la concepción tradicional de la Administración, por lo que invertir en las adecuaciones necesarias para lograr ese cambio de paradigma es imprescindible, de lo contrario estaría quedando rezagada (Muñoz, 2016: 26).

Todo lo cual crea las condiciones de posibilidad para una mejora en la concepción de la administración, tomando como referencia que el sistema democrático encuentra su fundamento en las instituciones y los acuerdos a que lleguen los gobernantes con los grupos de interés y la ciudadanía organizada, es decir, lo que se denomina la consensualidad política, evidenciada a través de audiencias públicas en las cuales esté presente la institucionalidad en cada parte del territorio nacional, en las cuales la comunidad interviene directamente, las instituciones muestran su potencialidad y se evita la congestión y el desgaste administrativo y judicial, por circunstancias tan efímeras como la incoación de peticiones, acciones constitucionales y legales. 
Angéliç Gavidia Pacheco, Eder Alberto Molina Araujo, Ingrid Julissa Illidge Correa, Leonardo de Jesús Díaz y Indira Yulieth Illidge Correa

Utilización eficaz de las tecnologías de información y comunicación en procesos sancionatorios y responsabilidad fiscal

\section{Conclusiones}

El Estado como garante de los deberes y derechos del ciudadano debe buscar la manera de satisfacer las necesidades de la población, quien espera en democracia que sus representantes delegados satisfagan sus necesidades. En este sentido, se busca que el Estado sea dinámico, ágil y eficaz en sus trámites de lo contrario se estarían afectan un conjunto de derecho; es por ello, que el legislador ha expedido normas como la Ley 1712 de 2014 que desarrollo el derecho fundamental al acceso a la información pública y el Modelo Integrado de Planeación y Gestión con sus dimensiones y componentes que buscan precisamente acercar más el Estado al ciudadano, que es su razón de ser en virtud de la teoría del contrato social.

Cuando se extienden las herramientas tecnológicas a la institucionalidad del Estado Colombiano se logra tener un mejor acercamiento con la ciudadanía, la cual podrá tener una expectativa positiva en la satisfacción de sus necesidades, siendo esta la esencia del Estado Social de Derecho. Por ello, los órganos de control fiscal territorial requieren mayor fortalecimiento, a través de recursos con destinación específica, en tecnología de la información aprovechando las oportunidades que se generaron a partir del Acto Legislativo 04 de 2019, del cual deben definirse medidas de fortalecimiento del control fiscal territorial a través de proyectos de ley que fomenten y masifiquen estas tecnologías de información, habida cuenta que se permita el ejercicio de una facultad administrativa (sancionatoria y de responsabilidad fiscal) que además de cumplir con los cometidos y finalidades de ejercicio de su objeto misional en los órganos de control, permita la satisfacción de necesidades a los ciudadanos en cumplimiento del Modelo Integrado de Planeación de Gestión.

En efecto, es importante que la administración pública se actualice a las herramientas tecnológicas, debido a que permite la eficiencia y efectividad administrativa en la generación de garantías a la ciudadanía, siendo importante que se coloque a todas las entidades públicas a tono con estas herramientas, no puede ser que haya entidades que no brinden dichas posibilidades, limitando de ésta forma el derecho a la publicidad y garantías ciudadanas, más aun cuando se habla de un Modelo Integrado de Gestión centrado en personas; por lo que en los procesos de responsabilidad fiscal y sancionatorios que llevan las contralorías territoriales deben utilizarse dichas herramientas en forma general, siempre que así lo haya solicitado o permitido tácitamente los involucrados o partes interesadas, esto sin duda colocará a tono dichas entidades públicas, que pareciera se hubiesen quedado en el tiempo, requiriendo de una inversión en materia de innovación tecnológica, tal como se le otorgó a la Contraloría General de la República. 


\section{Referencias Bibliográficas}

AIBAR, Eduardo. 2001. "Fatalismo y tecnología: ¿es autónomo el desarrollo tecnológico?” En: OUC. Disponible en línea. En: http://www.uoc.edu/ web/esp/art/uoc/0107026/aibar.html. Fecha de consulta: 04/21/2019.

ASAMBLEA NACIONAL CONSTITUYENTE. 1991. Constitución Política de Colombia. Gaceta Constitucional No. 116. Disponible en línea. En: https://www.corteconstitucional.gov.co/inicio/Constitucion\%20 politica\%2ode\%20Colombia.pdf. Fecha de consulta: 12/12/2019.

BIMBER, Bruce. 1994. Tres caras del determinismo tecnológico. Madrid, España.

CASTELLS, Manuel. 2001. La Galaxia Internet. Reflexiones sobre Internet, Empresa y Sociedad. Plaza y Jánes (Arete). Barcelona, España.

CONGRESO DE LA REPÚBLICA DE COLOMBIA. 2019. Acto Legislativo o4 de 2019 Congreso de la República. Disponible en línea. En: https://www.funcionpublica.gov.co/eva/gestornormativo/norma. php?i=100251\#: :text=Dictar\%2onormas\%20generales\%20para\%20 armonizar,la\%20vigilancia\%20y\%20control\%20de. Fecha de consulta: $12 / 12 / 2019$.

CONGRESO DE LA REPÚBLICA. 2009. Ley 1341. DO No. 47.426. Gaceta Oficial. Bogotá, Colombia.

CONGRESO DE LA REPÚBLICA. 2011. Ley 1437. DO No. 47.956. Gaceta Oficial. Bogotá Colombia.

CONGRESO DE LA REPÚBLICA. 2014. Ley 1712 de 2014. Disponible en línea. En: https://www.funcionpublica.gov.co/eva/gestornormativo/norma_ pdf.php?i=56882. Fecha de consulta: 12/12/2019.

CONSEJO DE ESTADO. 2011. Fallo 763. Radicado 2006-00036-oo. Sala de lo Contencioso

Administrativo. C.P. Gerardo Arenas Monsalve. Bogotá D.C.

CONSEJO DE ESTADO. 2012. Radicado Número (0300-06) Sala de lo Contencioso

Administrativo. C.P. Gerardo Arenas Monsalve. Bogotá D.C.

CORTECONSTITUCIONAL.1992.SentenciaT-582. M.P.Dr.EduardoCifuentes Muñoz. Disponible en línea. En: https://www.corteconstitucional.gov. co/relatoria/1992/T-582-92.htm. Fecha d consulta: 14/06/2018. 
Angéliç Gavidia Pacheco, Eder Alberto Molina Araujo, Ingrid Julissa Illidge Correa, Leonardo de Jesús Díaz y Indira Yulieth Illidge Correa

CORTECONSTITUCIONAL. 1993. SentenciaT-145. M.P.Dr. Eduardo Cifuentes Muñoz. Disponible en línea. En: https://www.corteconstitucional.gov. co/relatoria/1993/t-145-93.htm. Fecha d consulta: 14/o6/2018.

CORTECONSTITUCIONAL. 1996.Sentencia C-319.M.P. Dr. Vladimiro Naranjo Meza. Disponible en línea. En: http://legal.legis.com.co/document/ Index?obra=jurcol\&document=jurcol_7599204158d1fo34e0430a0101 51fo34. Fecha de consulta: 12/12/2019.

CORTE CONSTITUCIONAL. 2000. Sentencia C-662. M.P. Dr. Fabio Morón Díaz. Disponible en línea. En: https://www.redjurista.com/ Documents/corte_constitucional,_sentencia_de_control_de_ constitucionalidad_no._662_de_2000.aspx\#/. Fecha de consulta: 12/12/2019.

CORTE CONSTITUCIONAL. 2013. Sentencia C-012. M.P. Dr. Mauricio González Cuervo. Disponible en línea. En: http://www.suinjuriscol.gov.co/viewDocument.asp?id=20000532. Fecha de consulta: 12/12/2019.

CORTE CONSTITUCIONAL. 2013. Sentencia C-286. M.P. Dr. Nilson Pinilla Pinilla. Disponible en línea. En: https://www.funcionpublica. gov.co/eva/gestornormativo/norma_pdf.php?i=57028. Fecha de consulta: 12/12/2019.

GARCÍA GARCÍA, Jesús. 2014. “Gobierno abierto: transparencia, participación y colaboración en las administraciones públicas" En: INNOVAR. Revista de Ciencias Administrativas y Sociales. Disponible en línea. En: https://www.redalyc.org/pdf/818/81832222006.pdf. Fecha d consulta: 14/06/2018.

GUILLÉN CARAMÉS, J (2010) La administración electrónica: ¿Mito o realidad para los ciudadanos del siglo XXI? Disponible en línea. En: https://www. pwc.es/es/crecimiento-inteligente/assets/administracion-electronica. pdf. Fecha de consulta: o8/03/2021

HABERMAS, Jürgen. 1970. Hacia una sociedad racional. Editorial Faro. Barcelona, España.

LAGUADO GIRALDO, Roberto. 2003. “Actos Administrativos por medios electrónicos” En: Revista Vniversitas. Disponible en línea. En: https:// revistas.javeriana.edu.co/index.php/vnijuri/article/view/14842. Fecha de consulta: 15/04/2018.

MASUDA, Yoneji.1984. La sociedad informatizada como sociedad postindustrial. Fundación

MATTERLART, Armand. 2001. Historia de la sociedad de la información. Edición revisada y ampliada por el autor. Paidós. Barcelona, España. 
MINISTERIO DE COMUNICACIONES, Documento Conpes 3072. Disponible en línea. En: https://mintic.gov.co/portal/inicio/3498:Conpes-3072de-2000

MOYA GARCÍA, Rodrigo. 2008. "El Procedimiento Administrativo Electrónico en Chile" Tesis para optar al grado de Magister en Derecho Público. Universidad de Chile. Disponible en línea. En: http://repositorio.uchile. cl/tesis/uchile/2008/de-moya_r/pdfAmont/de-moya_r.pdf. Fecha de consulta: 15/04/2018.

MOYADO ESTRADA, Francisco. 2011. "Gobernanza y calidad en la gestión pública” En: Estudios Gerenciales. Disponible en línea. En: https://www. redalyc.org/pdf/212/21222706011.pdf. Fecha de consulta: 12-02-2019.

MUÑOZ YANDAR, Alba. 2016. "La implementación de las TIC en el procedimiento administrativo, como herramienta para garantizar el derecho de los asociados a relacionarse electrónicamente con la administración pública" En: Universidad Nacional de Colombia. Disponible en línea. En: https://repositorio.unal.edu.co/handle/ unal/59652. Fecha de consulta: 02/11/2019.

NIETO GARCÍA, Alejandro. 1994. Derecho administrativo sancionador, $2^{\mathrm{a}}$ edición. Editorial Tecnos S.A. Madrid, España.

PARRA QUIJANO, Jairo. 2004. Manual de Derecho Probatorio. Librería Ediciones del Profesional Ltda. Bogotá, Colombia.

SÁNCHEZ ACEVEDO, Marcos. 2014. Eficacia y validez del acto administrativo electrónico.

Una perspectiva en el derecho comparado entre Colombiay España. Universidad Católica de Colombia. Bogotá, Colombia.

SANTOFIMIO-GAMBOA, Jaime. 2011. Procedimientos administrativos y tecnología. Externado de Colombia. Bogotá, Universidad. Tecnos. Madrid, España. 
Vol. 39 N $^{\circ} 69$

Esta revista fue editada en formato digital y publicada en julio de 2021, por el Fondo Editorial Serbiluz, Universidad del Zulia. Maracaibo-Venezuela 